\title{
Calidad de servicio como elemento clave de la responsabilidad social en pequeñas y medianas empresas
}

\author{
Claudia Rojas-Martínez ${ }^{(1)}$, William Niebles-Nuñez ${ }^{(1)}$, Carlos Pacheco-Ruíz ${ }^{(1)}$, y Hugo G. Hernández-Palma ${ }^{(2)}$ \\ (1) Universidad de Sucre, Programa de Administración de Empresas, Sincelejo-Colombia. \\ (correo-e: claudia.rojas@unisucre.edu.co; william.niebles@unisucre.edu.co; carlos.pacheco@unisucre.edu.co) \\ (2) Universidad del Atlántico, Programa Administración de Empresas, Barranquilla-Colombia. \\ (correo-e: hugohernandezpalma@gmail.com)
}

Recibido Feb. 18, 2020; Aceptado Abr. 21, 2020; Versión final May. 12, 2020, Publicado Ago. 2020

\begin{abstract}
Resumen
El presente estudio analizó la calidad de servicio como elemento clave para cumplir con la responsabilidad social en pequeñas y medianas empresas (pymes). La metodología fue descriptiva, no experimental y de campo. La población fue de 50 pymes del sector logístico representadas cada una por un empleado. Los datos se recolectaron con cuestionarios con alternativas tipo Likert con una confiabilidad de 0.80 y 082 . Con el método de Pearson se percibió una correlación de 0.025. Es decir, aunque la calidad de servicio no sea óptima, pudieran cumplir con su responsabilidad social. Los datos fueron tabulados con estadísticas descriptivas a través de un proceso eficiente en la calidad de servicio. Las pymes asumen cumplir con su responsabilidad social puesto que ofrecen un servicio eficiente y efectivo para lograr los objetivos. Se concluye que la responsabilidad social depende en un nivel muy bajo de la calidad de servicio ofrecido por las pymes.
\end{abstract}

\section{Quality service as a key element of social responsibility in small and medium size enterprises}

\begin{abstract}
The present study focused on analyzing service quality as a key element to meet social responsibility in small and medium size enterprises (SMEs). The methodology was descriptive, not experimental, and field based. The population was 50 employees from 50 logistic sector SMEs. The data were collected with questionnaires with Likert alternatives with a reliability of 0.80 and 082 . Pearson's method perceived correlation was of 0.025 . This means that even if the quality of service is not optimal, they could fulfill their social responsibility. The data were tabulated with descriptive statistics through an efficient process in the quality of service. SMEs assume to fulfill their social responsibility since they offer an efficient and effective service to achieve their objectives. However, it is concluded that social responsibility depends at a very low level on the quality of service offered by SMEs.
\end{abstract}




\section{INTRODUCCIÓN}

En la actualidad, los métodos utilizados para gerenciar las empresas han ido evolucionando, ello producto de la globalización, pero además derivado del éxito alcanzado por los lineamientos establecidos desde las organizaciones japonesas, estas estructuradas y sustentadas en los preceptos de la filosofía de la calidad. De acuerdo con ello, la mayoría de las organizaciones a nivel mundial están orientadas a la prestación de un servicio de alta calidad, convirtiéndose esta en una de las tareas más importantes de ejecutar, pero además que se ha transformado en un proceso arduo y difícil de aplicar en las organizaciones que se dedican al servicio en general. Se sustenta que la globalización ha contribuido con un proceso de internacionalización económica, involucrando el crecimiento del comercio además de la inversión internacional, dando pie a la expansión del capitalismo como modelo económico y modo de producción en el ámbito mundial; sumado a esto, se refleja en la creciente importancia de la estructura financiera, en el aumento del saber, el crecimiento de la transnacionalización de la tecnología basadas en el conocimiento y el ascenso de corporaciones multinacionales.

En este orden de ideas, las empresas han ido logrando vincularse a una nueva etapa de desafíos, cambios y transformaciones, orientadas a la búsqueda de la calidad del servicio, para lograr mantenerse a la par de sus competidores a lo largo del tiempo, ello implica que cada trabajador asuma un alto grado de responsabilidad en el desarrollo de esta. Es así como los elementos como la efectividad, productividad, competitividad, así como la eficacia organizacional son variables fundamentales, razón por la cual se hace necesaria la aplicación de métodos o técnicas que permitan optimizar el desenvolvimiento de los procesos de producción; así como la administración en las organizaciones, en virtud de mejorar las actividades laborales optimizando los procesos de trabajo. En este sentido hoy en día, la calidad del servicio es un sistema altamente empleado por todas las organizaciones, las cuales, según Durán et al., (2017), buscan posicionamiento, liderazgo, pero además principalmente la satisfacción de sus consumidores, lo cual es un factor determinante para toda empresa prestataria de un servicio, tomando mucha relevancia al respecto.

Dichas organizaciones han reconocido que sólo ofreciendo un servicio de excelente calidad según Kerin y Hartley (2015), es como conquistarán esa satisfacción, en este ámbito buscan obtener ese posicionamiento, el cual podría considerarse como base fundamental para el crecimiento y fortalecimiento en el mercado donde se desenvuelve. Bajo este contexto, la calidad de servicio en las organizaciones radica en orientar a los clientes en lograr cumplir con una necesidad, y además facilitarle el cumplimiento de sus expectativas individuales, por otra parte, la atención brindada debe ser buena y personalizada. Dentro de este marco, Pride y Ferrell (2018) señalan que la prestación de servicios de alta calidad es una de las tareas más importantes y difíciles que afronta cualquier organización de servicios, además resalta que tal suministro de manera consistente de un servicio de alta calidad es muy difícil, se fundamenta en el hecho que todos los consumidores han experimentado ejemplos de un servicio deficiente, se piensa que los servicios forman parte de una analogía, la cual constituye un simple instrumento de racionalización.

Por lo antes expuesto, es necesario optimizar los recursos en las empresas, con el fin de alcanzar las metas y objetivos propuestos, con eficiencia y eficacia, como consecuencia de un proceso de globalización e intercambio económico que obligan a las organizaciones a diferenciase de sus competidores. En este sentido, De la Hoz et al (2020), sostienen que a medida que las organizaciones confrontan las nuevas competencias exigidas por los mercados a nivel Mundial, la apertura y el progreso de tecnología requieren con mayor disposición la permanencia de un capital humano apto y disponible en lugares y momentos específicos. Aunado a esto, el capital humano, posee una función relevante para el cumplimiento de los objetivos planeados por la gerencia, ellos requieren de una planificación equilibrada que considere todos los recursos disponibles y necesarios para la empresa, Moreno et al (2018). Entre ellos, los recursos humanos, tecnológico, así como el área de trabajo en forma integrada y sistémica, de esa manera satisfacer las necesidades de los empleados, al mismo tiempo debe traducirse en un servicio óptimo al cliente, en este caso como se generan los procesos de responsabilidad social interna. A este respecto, Koontz y Weihrich (2012), consideran que está ampliamente demostrado que la responsabilidad social interna puede transformarse en una eficiente estrategia para la gestión organizacional, donde sus dimensiones están vinculadas con la gestión organizacional, así como la fusión de la ética corporativa, recolocación, reconocimiento interno, mejoramiento del ambiente de trabajo.

En este ámbito se hace manifiesto que la responsabilidad social de las empresas va más allá de obtener utilidades, haciéndose necesario mantener un equilibrio entre el bienestar empresarial y de la sociedad, bajo este contexto, la supervivencia de las organizaciones depende del apoyo y respeto obtenido de la comunidad donde se desenvuelve, de allí la necesidad del intercambio equitativo, entre ambos integrantes, por ello las organizaciones deben ser socialmente responsables, combinando en un forma equilibrada los aspectos legales, éticos, morales y ambientales. 


\section{RESPONSABILIDAD SOCIAL}

Robbins y Jugde (2017), consideran a la responsabilidad social como la actitud voluntaria de la empresa dirigida a satisfacer las demandas sociales de los distintos agentes relacionados, derivados de los efectos que provoca la actividad empresarial sobre estos. Se sugiere que cada empresa, en función de sus valores y compromisos sociales, debe definir el campo de actuación de su responsabilidad social. Atendiendo a estas consideraciones las organizaciones, deben asumir cierto grado de responsabilidad social, incorporando a la planificación objetivos socioeconómicos vinculados a los grupos que intervienen directa o indirectamente en su desarrollo, intentando buscar un equilibrio entre las funciones económicas y sociales. En tal sentido, cualquier acción llevada a cabo por una empresa afecta al bienestar de sus grupos de interés. Por ende, una determinada estrategia puede aumentar el bienestar de algunos y causar daño a otros. Por tanto, la responsabilidad social ha de incorporarse a la estrategia de la empresa, debe estar íntimamente ligada a su proceso de planificación estratégica y a su correspondiente control, orientado a fomentar la calidad de servicio.

Para Drucker (1965), citado por Koontz y Weihrich (2012), las líneas básicas que enmarcan las responsabilidades sociales son: un volumen adecuado de beneficios, el mismo señala como primer deber orientándola a sí misma, así como hacia los hombres que en ella trabajan, el cual en su opinión está seguido inmediatamente por la necesidad de crecimiento de la capacidad de producir y crear riqueza. En el mismo orden de ideas, los mismos Koontz y Weihrich (2015), expresan que la responsabilidad social se asocia con las relaciones, obligaciones y deberes generalmente aceptados que las organizaciones tienen con la sociedad, involucrando la integración voluntaria, por parte de las empresas, de las preocupaciones sociales y medioambientales en sus operaciones comerciales y en las relaciones con sus interlocutores, además sustenta que se ha producido un cambio en el paradigma de funcionamiento de las empresas, pasando de un fin exclusivamente dirigido a los resultados económicos, a un triple objetivo: lo económico, lo social y lo medioambiental, generando como consecuencia o resultado una calidad de servicio óptimo, tanto para el cliente interno como externo.

Como consecuencia de los dos puntos anteriores, la estrategia y actividad de la empresa tienen que adaptarse a los cambios producidos en el ambiente externo; asimismo, los comportamientos o actividades de la empresa deben cumplir los requisitos de sostenibilidad y de interrelación con los grupos de interés, a los cuales les ofrece servicio. Bajo este contexto, las organizaciones socialmente responsables pueden obtener una posición sólida respecto a su competencia al poseer una serie de ventajas competitivas en el mercado, al establecer su pertinencia con el entorno. Asumiendo cierto compromiso por las repercusiones de sus actividades sobre sus miembros y sobre la colectividad en general, ello incide en el desarrollo productivo de la empresa.

En este orden de ideas, Parra, et al., (2019) sostienen que las organizaciones se muestran partidarias de dedicar esfuerzos a programas orientados a mejorar su entorno físico - social, generando estrategias de atención al empleado, pero además promoviendo el crecimiento y desarrollo de las comunidades externas, ello influye positivamente en la opinión pública, en pro de incrementar las ventas e indirectamente los beneficios a largo plazo, sin costos añadidos. En este sentido, la responsabilidad principal de las empresas está orientada a generar beneficios, que contribuyan al logro de objetivos sociales y medioambientales, integrando la responsabilidad social como inversión estratégica en el núcleo de su estrategia empresarial, sus instrumentos de gestión y sus actividades. En este orden de ideas, en el libro verde de la Comisión Europea (2002) se sugiere que la actividad empresarial genera múltiples riesgos de producción de daños a los trabajadores, asociados a las condiciones sociales y materiales en la cual se realiza el trabajo, estos pueden afectar el estado de bienestar de las personas en forma negativa.

Asimismo, las empresas deben generar acciones responsables hacia los empleados, promoviendo un ambiente laboral positivo: permitirles el equilibrio entre trabajo, descanso, vida de familia; propiciar espacios de formación, educación, así como de crecimiento personal; respetar creencias religiosas y diferencias culturales; procurarles condiciones de ambiente saludables, agradable, ofrecerles las herramientas adecuadas; además realizar campañas de salud, prevención de vicios, protección al medio ambiente; estimular el deporte, entre otros. Perreault Cannon y McCarthy (2013). Por otro lado, las organizaciones deben orientarse a disminuir los riesgos que corre el empleado en el área de trabajo, para promover: la mejora continua, compromiso con el personal, eficiencia, y competitividad. De manera que puedan ser contempladas como elementos estratégicos en la gestión global de cualquier empresa. Con respecto a este último punto, este conjunto de elementos se les pueden asociar con la propuesta de Zeithaml, et al., (2017), quienes definen la calidad de servicio como el componente primordial de las percepciones del cliente, y ese servicio es concebido como parte del cumplimiento de la responsabilidad social de una empresa.

Para García et al., (2017), el objetivo de toda empresa es conseguir resultados favorables, lo cual se presenta con la diferenciación; ser diferente, en eso consiste la estrategia competitiva, así como de su 
proceso estratégico, por ende en los servicios la calidad es el elemento que predomina en las evaluaciones de los clientes, en los casos que ofrecen servicios en combinación con un producto físico permite determinar la satisfacción del cliente, lo cual determina el cumplimiento de la responsabilidad social, según lo planteado por Díaz, et al., (2017). Es decir, la calidad de servicio se determina por las diferentes percepciones del cliente de acuerdo con su necesidad, es allí donde deben coincidir los mejores atributos al momento que el cliente demande un producto o servicio, para satisfacer dicha necesidad en el momento preciso. Pues bien, según Paz, Harris y García (2015), la calidad del servicio es el nivel de excelencia que la empresa ha escogido para satisfacer a sus clientes. Clientes que, a su vez, imponen el nivel del servicio que suele alcanzar toda empresa, a través de necesidades y expectativas. Por tanto, solo será competitiva la empresa que adopte los mejores criterios de calidad y ofrezca la mayor gama de servicio al cliente de acuerdo con sus necesidades, Jiménez et al., (2018).

En este mismo orden de ideas, Jiménez, et al., (2018), las organizaciones que responden a los múltiples retos en los diversos ámbitos a nivel Nacional, han abordado con eficiencia, un conjunto de cambios internos; Bravo, et al (2017), tales como implantación de modelos gerenciales que involucren al personal hacia el enlace de las metas establecidas, con lo cual contribuyan al mantenimiento de la organización, así como el uso eficiente de los recursos tanto económicos como humanos haciendo frente en un mercado altamente competente. Entre las organizaciones que se encuentran inmersas en estos procesos, están las del sector logístico, el operador logístico es un colaborador estratégico de las empresas productoras y comercializadoras de materias primas, insumos y productos terminados (o de servicios), quien, diseña y desarrolla integralmente los procesos de las fases de su cadena de abastecimiento (transporte internacional, aprovisionamiento, transporte terrestre, almacenaje, distribución, maquila, incluso trámites de legalización y documentación de las mercancías).

Asimismo, Martínez, et al., (2017), manifiestan que el operador logístico gestiona, el desarrollo de las operaciones, empleando de forma eficiente y segura infraestructura física, tecnología, sistemas de información y talento humano, que pueden ser suministrados por el cliente o ser propios del operador logístico. Lo que a su vez, implica una forma distinta de trabajo puesto que mide y revisa la eficiencia con la que se realizan las distintas actividades en estas Pymes donde procesos, productos y servicios se ofrecen con calidad estable, tendencias a la mejora continua y trayendo además como resultado un incremento en la productividad, mejora de su situación financiera y diferenciación de su competencia lo cual puede ser alcanzado a través de la certificación como Operador Económico Autorizado (OEA), los cuales son empresas que en el marco de sus actividades operativas y profesionales, efectúan actividades reguladas por la legislación aduanera.

En los actuales momentos algunos de los problemas a los que se enfrentan las pymes operadoras del sector logístico tienen que ver con las políticas gubernamentales, infraestructura vial, costos de los combustibles, inserción en los tratados de libre comercio, atrasos en los tiempos de entrega, daños en la mercancía, desconocimiento de las normas internacionales y poca experiencia para realizar trámites en aduanas. Por lo que en diversas ocasiones los clientes consideran alguna deficiencia en la calidad del servicio recibido. Lo que conlleva a pensar que estas pymes del sector operadores de carga de la región Caribe, muestran una problemática que implica tanto aspectos internos como aspectos externos, los cuales impactan en la competitividad de estas Pymes. Dada esta situación, se justifica que la gran variedad de ofertas en el sector y las mejoras tecnológicas hacen que cada vez sea más difícil percibir diferencias entre los productos; de esta forma, el servicio al cliente juega un rol preponderante en cuanto a la diferenciación y competitividad de las empresas en los mercados. Atendiendo a las consideraciones anteriores, los investigadores se plantearon como objetivo analizar la calidad de servicio fomentada desde la responsabilidad social interna en Pymes.

\section{OTROS ANTECEDENTES}

Hay una serie de antecedentes adicionales los que se hace necesario detallar para documentar con mayor profundidad la temática los cuales son: i) elementos de la calidad de servicio; ii) necesidades del cliente; iii) expectativas del cliente; y iv) factores de la responsabilidad social. Cada uno de estos aspectos son expuestos a continuación.

\section{Elementos de la calidad de servicio}

En la actualidad, según García, et al., (2017), los clientes se han vuelto tan conscientes de los que les debe brindar un servicio, por ello sus expectativas sobre el servicio se han elevado, a tal punto que ya no agradecen un servicio superficial. Esto al menos indica cuan generalizados se han vuelto los programas de servicio al cliente; sin embargo, además de estas expectativas cada vez mayores, parece haber un escepticismo creciente en cuanto a uno de ellos, los clientes son los verdaderos beneficiarios. En virtud de ello, las nuevas tendencias, sugieren lo que los clientes quieren ahora es profundidad (sustancia) en el 
servicio, lo cual les prometen: una plena percepción del confort, además de gusto; esto proviene de algo más que superficialidades. Bajo este contexto, los elementos básicos de la calidad del servicio son las siguientes:

\section{Necesidades del cliente}

Stanton, et al., (2012) explican que surge una necesidad cuando es susceptible de satisfacerse y que por medio del consumo se vuelve lo bastante fuerte como para motivar al sujeto a satisfacer la necesidad. Como se puede observar los autores anteriormente citados, coinciden en que la necesidad es una fuerza que impulsa a realizar alguna acción que satisfaga la misma. Según Kotler (2017), las empresas que tienen más éxito son aquellas capaces de proporcionar al cliente, el servicio esperado; o superar sus expectativas o necesidades. Estas empresas practican de manera consciente o inconsciente lo que se conoce como la total satisfacción del cliente (TSC), la cual se refiere a cubrir solucionar el problema del cliente, además de satisfacer sus necesidades, y hacer que el cliente sienta que ha recibido un servicio de verdadera calidad, dichas necesidades tienen también sus diferencias.

\section{Expectativas del cliente}

Otro de los elementos son las expectativas del cliente, se puede afirmar que las personas en general ven lo que esperan ver, basado en su realidad, sustentado en su experiencia anterior o en un medio preacondicionado. Pueden considerarse como una esperanza, un prospecto, entre otros. Algunos profesionales del marketing del servicio han concluido que el cliente tiene varias expectativas del servicio. Se han detectado dos niveles en el tema de las expectativas. Según Zeithaml, et al., (2017), el primer nivel puede denominarse servicio deseado, es la combinación de lo que el cliente considera que puede ser, contra su pensamiento el servicio. El segundo nivel se llama servicio adecuado, es el nivel del servicio aceptado por el cliente. El nivel de servicio que los clientes consideran aceptable es afectado por otros determinantes, por lo general de este tipo de influencias es de corto plazo y tiende a fluctuar más que los factores relativamente estables que influyen en el servicio deseado. Ellos se explican a continuación, según la posición de: Zeithaml, et al., (2017), como puede apreciarse en la figura 1, denominada servicio adecuado.

Intensificadores transitorios del servicio: son factores individuales
temporales y usualmente a corto plazo que provocan que el cliente sea más consciente de la necesidad del servicio.

Percepción de las alternativas del servicio: son los proveedores de los cuales el cliente puede obtener el servicio. Si el cliente puede elegir entre múltiples proveedores para un mismo servicio o si pueden prestarse el servicio a sí mismo.

Auto percepción del papel del cliente en el servicio: se define como las percepciones de los clientes acerca del grado de influencia que ejercen sobre el nivel de servicio que reciben. En otras palabras las expectativas del cliente se modelan, en cierta medida, por el grado de desempeño de su papel en la prestación del servicio que creen tener.

Servicio predicho, el nivel de servicio que los clientes creen que probablemente recibirán. Este tipo de expectativas de servicio se puede observar como las proyecciones que realizan los clientes acerca de lo que puede suceder durante una transacción o intercambio inminente.

Fig. 1. Factores del servicio adecuado. Adaptado de Zeithaml, Bitner y Gremler (2017)

Posteriormente se habla del servicio deseado, involucra las influencias que afectan de manera importante el nivel de servicio deseado son las necesidades personales y las filosofías del servicio. Las necesidades personales, es decir, los estados y condiciones esenciales para el bienestar físico y psicológico del cliente, son elementos fundamentales que dan forma al nivel de servicio deseado. Por otra parte, los intensificadores permanentes del servicio son factores individuales y estables que exaltan la sensibilidad de los clientes ante el servicio. Las expectativas derivadas del servicio son de los componentes más importantes que se presenta cuando las expectativas de los clientes son dirigidas por otra persona o grupo 
de personas. Otro intensificador permanente del servicio es la filosofía personal del servicio, es decir, la actitud genérica que subyace en los clientes acerca del significado de servicio y la conducta apropiada de los proveedores de servicio.

\section{Factores de la responsabilidad social}

La responsabilidad social está relacionada con diferentes agentes los cuales los cuales tienen su influencia, de acuerdo con la realidad en la cual se desenvuelve, Certo, et al., (2007), en este sentido, una empresa responsable socialmente establece como principal estandarte en su cultura organizacional, la ética, la moral, los principios cívicos, pero también los ciudadanos, así como todo lo referente a sus valores, discriminados según los intereses de la institución. Aunado a ello, Robbins y Coulter (2015), consideran que los factores a favor de los argumentos principales de responsabilidad social son: 1) Expectativas públicas; 2) Utilidades a largo plazo; 3) Obligación ética; 4) Imagen pública; 5) Mejor entorno; 6) Oposición a reglamentos gubernamentales adicionales; 7) Equilibrio entre responsabilidad y poder; 8) Intereses de los accionistas; 9) Posesión de recursos; y 10) Superioridad de prevención sobre remedios.

El primer factor es que las expectativas sociales de los negocios han incrementado de manera espectacular desde la década de los sesenta. La opinión pública ahora apoya que los negocios tengan metas tanto sociales como económicas. El segundo se basa en que los negocios socialmente responsables tienden a tener utilidades a largo plazo mejor aseguradas. Este es el resultado normal de mejores relaciones con la comunidad y una mejor imagen de negocios que conlleva el comportamiento responsable. Según el tercero una firma de negocios puede y debe tener conciencia social. Los negocios deben ser socialmente responsables porque las acciones responsables son las correctas para su propio beneficio. Con respecto al cuarto factor Padilla, et al., (2017), estipulan que las firmas buscan resaltar su imagen pública para obtener mayores ventas, mejores empleados, otros beneficios. Dado que el público considera las metas sociales importantes, los negocios pueden crear una imagen pública favorable al perseguir metas sociales. El quinto, se basa en que la participación del negocio puede contribuir a resolver problemas sociales difíciles ayudando a crear una mejor calidad de vida y una comunidad más deseable a la cual atraer y mantener empleados capacitados.

Cabe destacar que con el sexto los reglamentos gubernamentales agregan costos económicos y restringen la flexibilidad de decisiones de la gerencia. Al convertirse en socialmente responsables, los negocios pueden esperar tener menos reglamentos gubernamentales. El séptimo comprende que los negocios tienen una buena cantidad de poder en la sociedad. Se requiere una cantidad de responsabilidad igualmente importante para equilibrarlas. Sobre esto último Parody et al., (2018), sostienen que cuando el poder es significativamente mayor que la responsabilidad, el desequilibrio fomenta un comportamiento irresponsable por parte de los negocios, el cual opera en contra del bienestar público. Asimismo, según el octavo factor, la responsabilidad social mejorará el precio de las acciones de un negocio a largo plazo. El mercado de valores contemplará a la compañía socialmente responsable como menos riesgosa y abierta a críticas públicas.

Siguiendo con el noveno, las organizaciones tienen recursos financieros, técnicos especializados y talento gerencial que puedan apoyar proyectos públicos y de beneficencia que necesiten asistencia. Y, por último, el décimo trata de que los problemas sociales siempre deberán enfrentarse en algún momento. Los negocios deben actuar antes de que estos problemas se vuelvan más serios y costosos en su corrección, alejando la energía de la gerencia del cumplimiento de sus metas de producir bienes y servicios. De acuerdo a los factores antes expuestos, Díaz, et al., (2019), sostienen que se pone de manifiesto que las empresas a medida que asumen de una manera positiva su responsabilidad social, su futuro se verá asegurado entre otras cosas por el apoyo mismo del entorno, el cual percibe las buenas acciones de la organización, en este sentido en las empresas del sector logístico, estos factores se generan en forma continua, a pesar de no lograr cubrir en un cien por ciento las expectativas de su personal, en función de los materiales manipulados.

\section{METODOLOGÍA}

En este estudio, se llevó a cabo una investigación cuantitativa de enfoque epistémico positivista, catalogada como aplicada por su propósito; de alcance descriptivo; de diseño no experimental transeccional. Para recolectar la información se definió como población a 50 Pymes del sector logístico representadas cada una por 1 empleado, la muestra fue de tipo censal. La técnica implementada para el levantamiento de información fue la observación mediante encuesta, a través de un cuestionario simple para cada variable con escala tipo Likert, este instrumento fue diseñado por los investigadores quienes se sustentaron en los indicadores de cada variable, estableciendo 3 ítems por cada uno de ellos, con relación al contenido teórico, se constituyeron 27 preguntas, para cada instrumento. Para lograr el proceso lógico se establecieron 
diferentes alternativas tipo escala likert para cada instrumento, el referido a la calidad de servicio con escalas de: siempre, casi siempre, a veces, casi nunca y nunca; mientras que el referido a la responsabilidad social usa una escala de: totalmente de acuerdo, de acuerdo, neutral, en desacuerdo y totalmente en desacuerdo. Posterior a su diseño, los instrumentos fueron sometidos a la validez de contenido bajo el juicio de expertos, seleccionando 7 expertos: 5 especialistas en gerencia y servicio, y 2 en el área de metodología; en virtud de verificar la coherencia, pertinencia y adecuación de las preguntas con relación al objetivo que se quería lograr. Ambos cuestionarios fueron validados por los 7 expertos, quienes hicieron recomendaciones sobre la redacción de algunos ítems. Posterior a la validación se procedió a identificar la confiabilidad del instrumento, para ello se aplicó una prueba piloto a una muestra tomada al azar, representada por 10 sujetos de 10 empresas con características similares a la población objeto de estudio en un municipio aledaño a Barranquilla.

Luego de ello se calculó la confiabilidad utilizando el coeficiente de Alfa Crombach, el cual es utilizado para instrumentos con diferentes alternativas de respuesta tipo escala likert, donde se obtuvo 0,80 (para el cuestionario de calidad de servicio) y 0,82 (para el de responsabilidad social) de confiabilidad, lo cual indica que son confiables. Consecutivamente para facilitar el desarrollo de los resultados se realizó el análisis de datos confrontando las inclinaciones de las repuestas presentadas en frecuencias absolutas (FA) y frecuencias relativas (FR), con base en estadísticas descriptivas de tendencia central como la media, moda, mediana y la desviación estándar. En este sentido, los resultados de este análisis fueron tabulados en tablas independientemente para su posterior interpretación lo que permitió obtener un diagnóstico de la situación o problemática planteada y establecer las conclusiones y recomendaciones pertinentes al caso de estudio además de darle respuesta al objetivo general de esta investigación. Para finalizar se calculó la correlación entre las variables a través del método de Spearman dado que ambas variables se han medido a través de una escala estadística ordinal. Para ello se computaron los datos arrojados y se les designo la letra "X" para la variable Calidad de servicio y la letra "Y" para la variable Responsabilidad. El coeficiente de Spearman es una medida de correlación para variables en un nivel de medición ordinal, ellos en virtud de que los individuos de la muestra se ordenaron por rangos.

\section{RESULTADOS}

La Tabla 1, contiene los resultados asociados a la dimensión elementos de la calidad de servicio, la misma está constituida por dos subdimesiones: necesidad del cliente y expectativa del cliente; a este respecto los resultados indican un alto nivel en la calidad de servicio en función de los promedios arrojados, generando un total para la dimensión 3.6 de promedio.

Tabla 1. Elementos de la calidad de servicio

\begin{tabular}{|c|c|c|c|c|c|c|c|c|c|c|c|c|c|c|c|c|}
\hline $\begin{array}{c}\text { Sub } \\
\text { Dimensión }\end{array}$ & Indicador & Ítems & & & & Iternati & vas $C$ & le Res & bues & & & & & & & \\
\hline Frecuenc & as de respues & tas & Sier & mpre & & $\begin{array}{l}\text { asi } \\
\text { npre }\end{array}$ & & eces & & $\begin{array}{l}\text { asi } \\
\text { nca }\end{array}$ & & nca & & Pron & nedio & \\
\hline & & & FA & FR & FA & FR & $\mathrm{FA}$ & FR & FA & FR & FA & FR & Ítem & indic & sub & Dim \\
\hline & & 1 & 42 & $84 \%$ & 2 & $14 \%$ & 4 & $8 \%$ & 2 & $4 \%$ & 0 & $0 \%$ & 4,68 & & & \\
\hline & $\begin{array}{l}\text { Filosofía del } \\
\text { servicio }\end{array}$ & 2 & 43 & $86 \%$ & 1 & $2 \%$ & 3 & $6 \%$ & 3 & $6 \%$ & 0 & $0 \%$ & 4,68 & 4,7 & & \\
\hline Necesidad & & 3 & 41 & $82 \%$ & 3 & $6 \%$ & 4 & $8 \%$ & 2 & $4 \%$ & 0 & $0 \%$ & 4,66 & & & \\
\hline & & 4 & 40 & $80 \%$ & 3 & $6 \%$ & 7 & $14 \%$ & 0 & $0 \%$ & 0 & $0 \%$ & 4,56 & & & \\
\hline & $\begin{array}{c}\text { Necesidades } \\
\text { personales }\end{array}$ & 5 & 45 & $90 \%$ & 3 & $6 \%$ & 2 & $4 \%$ & 0 & $0 \%$ & 0 & $0 \%$ & 4,82 & 4,5 & & \\
\hline & & 6 & 32 & $64 \%$ & 7 & $14 \%$ & 4 & $8 \%$ & 3 & $6 \%$ & 4 & $8 \%$ & 4,20 & & & \\
\hline & & 7 & 11 & $22 \%$ & 4 & $8 \%$ & 5 & $10 \%$ & 3 & $6 \%$ & 27 & $54 \%$ & 2,38 & & & 0,0 \\
\hline & $\begin{array}{l}\text { Servicio } \\
\text { deseado }\end{array}$ & 8 & 41 & $82 \%$ & 3 & $6 \%$ & 1 & $2 \%$ & 2 & $4 \%$ & 3 & $6 \%$ & 4,54 & 3.8 & & \\
\hline & & 9 & 40 & $80 \%$ & 3 & $6 \%$ & 3 & $6 \%$ & 4 & $8 \%$ & 0 & $0 \%$ & 4,58 & & & \\
\hline & & 10 & 15 & $30 \%$ & 20 & $40 \%$ & 15 & $30 \%$ & 0 & $0 \%$ & 0 & $0 \%$ & 3,40 & & 0.5 & \\
\hline & Servicio & 11 & 21 & $42 \%$ & 15 & $30 \%$ & 9 & $18 \%$ & 5 & $10 \%$ & 0 & $0 \%$ & 3,16 & 32 & & \\
\hline & & 12 & 13 & $26 \%$ & 23 & $46 \%$ & 12 & $24 \%$ & 2 & $4 \%$ & 0 & $0 \%$ & 2,92 & & & \\
\hline
\end{tabular}


La subdimensión necesidades del cliente tuvo un promedio de 4.6, siendo su tendencia muy alta con respecto al baremo de interpretación, este aspecto estuvo integrado por dos indicadores; el primero denominado filosofía del servicio arrojó una media de 4,7 a partir de los ítems 1, 2 y 3, donde los mayores puntajes se ubican en la categoría siempre, para el ítem 1 (el servicio que le ofrecen al cliente es rápido) un $84 \%$, para el ítem 2 (el servicio ofrecido por parte de los empleados de esta oficina es preciso) un 86\%, asimismo, para el ítem 3 (la impresión que causa el personal al cliente es muy positiva) un $82 \%$, en consecuencia se infiere que la filosofía de servicio es un elemento clave en sector logístico. Por otro lado, el indicador necesidades personales logró un promedio de 4.5 y sus tendencias indican el puntaje más alto en la opción siempre con un $80 \%$ en el ítem 4, con respecto a promover un ambiente agradable en las oficinas. En cuanto al ítem 5 el puntaje más alto fue del $90 \%$ en la opción siempre, afirmando que la oficina posee las condiciones necesarias para brindar un excelente servicio. Finalmente, el ítem 6 presenta su puntaje en la opción siempre con un $64 \%$ con lo que se infiere que es importante la celeridad en la entrega del servicio.

Seguidamente se presenta las tendencias en la subdimensión expectativas del cliente cuyo promedio se ubicó en 3,5, indicando una alta identificación en cuanto a sus indicadores. El primero de ellos "el servicio deseado" alcanzó un promedio de 3.8. Bajo este contexto se agruparon los ítems 7, 8 y 9, que presentan una variación de las posiciones, dado que en el ítem 7, el puntaje más alto fue el $54 \%$ en la categoría nunca, exhibiendo entonces que la población objeto de estudio, consideró que el cliente no recibe diferentes opciones en el momento de solicitar un servicio en las oficinas del servicio al cliente. Un $22 \%$ consideró la opción siempre, un $10 \%$ a veces, un $8 \%$ casi siempre y un $6 \%$ casi nunca.

Asimismo, el ítem 8 presenta el mayor puntaje en la categoría siempre con un $82 \%$ de la población, determinando que en la empresa existen alternativas y variedad en los servicios, y posteriormente el ítem 9 , vincula un $80 \%$ en la opción siempre, que estipula que las empresas del sector se diferencian entre sí por las diversas opciones de productos y servicios. En cuanto al indicador servicio adecuado, el ítem 10 muestra la mayor tendencia orientada hacia el casi siempre con un $40 \%$, además la tendencia positiva se apoya en la categoría siempre vinculado a un 30\% de la población, por lo que se estima que el servicio ofrecido por el personal es acorde a lo esperado por el cliente. Por otro lado, el ítem 11 involucra a un $42 \%$ con la categoría siempre, y añadiendo que un 30\% está ubicado en la categoría casi siempre, la información lograda permite inferir que los clientes asisten en forma regular a la empresa para solicitar sus servicios. Seguidamente el ítem 12 muestra que el $46 \%$ optó por la categoría casi siempre, apoyado en la categoría siempre con $26 \%$; cuyos resultados permiten inferir que la población objeto de estudio consideró que la relación del personal con el cliente promueve el logro de las metas. Según Kotler (2017) las empresas que tienen más éxito son aquellas capaces de proporcionar al cliente, el servicio esperado; o superar sus expectativas o necesidades. Refieren Zeithaml, et al., (2017), que para poder entender mejor el concepto y darle una definición más clara y profunda de las expectativas, con el propósito de comprenderlas, medirlas y administrarla.

Por otro lado, la Tabla 2, muestra los datos asociados a la dimensión factores de la responsabilidad social, constituido por cinco indicadores, el primero del segundo cuestionario realizado está referido a las expectativas públicas, en el cual se pretendió conocer con el ítem 1 si la empresa le brinda la seguridad en cuanto a interés por servirle de una manera incondicional. Asimismo, el ítem 2 "ejecuta actividades para fomentar el proceso de integración del empleado", por otra parte, el ítem 3 sobre si realiza campañas para mejorar su entorno. En este ámbito la mayoría de la población consideró que esto se ejecuta en la empresa, el 85,4\% de los encuestados estuvo totalmente de acuerdo, el 5,1\% asumió estar de acuerdo y mientras un $6 \%$ se mostró indiferente, no obstante, solo un $3,4 \%$ estuvo en desacuerdo.

Tabla 2. Factores de la responsabilidad social

\begin{tabular}{|c|c|c|c|c|c|c|c|c|c|c|c|c|}
\hline \multirow{3}{*}{ Indicadores } & \multirow{3}{*}{$\begin{array}{c}\text { Ítems } \\
\text { Agrupados }\end{array}$} & \multicolumn{10}{|c|}{ Alternativas de respuestas } & \multirow{3}{*}{ Promedio } \\
\hline & & \multicolumn{2}{|c|}{ TD } & \multicolumn{2}{|c|}{ DA } & \multicolumn{2}{|c|}{ NN } & \multicolumn{2}{|c|}{ ED } & \multicolumn{2}{|c|}{ TD } & \\
\hline & & FA & FR & FA & $\mathrm{FR}$ & FA & FR & FA & FR & FA & FR & \\
\hline $\begin{array}{l}\text { Expectativas } \\
\text { públicas }\end{array}$ & $1,2,3$ & 100 & 85,4 & 6 & 5,1 & 7 & 6 & 4 & 3,4 & 0 & 0 & 4,7 \\
\hline Obligación ética & $4,5,6$ & 90 & 77 & 13 & 11,1 & 6 & 5,1 & 4 & 3,4 & 4 & 3,4 & 4,5 \\
\hline Imagen pública & $7,8,9$ & 72 & 62 & 9 & 7 & 7 & 6 & 6 & 5,1 & 23 & 20 & 3,9 \\
\hline Mejor entorno & $10,11,12$ & 25 & 21,3 & 25 & 21,3 & 26 & 22,2 & 25 & 21,3 & 6 & 5,1 & 3,2 \\
\hline $\begin{array}{l}\text { Posesión de } \\
\text { recursos }\end{array}$ & $13,14,15$ & 10 & 9 & 18 & 15,3 & 37 & 32 & 10 & 9 & 42 & 36 & 2,5 \\
\hline
\end{tabular}


Luego el indicador obligación ética está orientado a demostrar si ejecuta acciones para el crecimiento personal del empleado con el ítem 4, a la mejora de los espacios físicos en el lugar de trabajo con el ítem 5, además el ítem 6 expresa si se orientan actividades laborales, en función de la calidad de vida del empleado. A lo cual contestaron de la siguiente manera: un $77 \%$ consideró estar totalmente de acuerdo, un $11,1 \%$ de acuerdo, el $5,1 \%$ se mantuvo indiferente, mientras que el restante $6,8 \%$ se dividió en partes iguales mitades asumiendo estar en desacuerdo y totalmente en desacuerdo, con respecto a las medidas estadísticas se obtuvo para el promedio un 4,5.

Otro elemento evaluado fue la Imagen pública, cuya tendencia se presenta hacia aspectos positivos en virtud de que el $62 \%$ manifestó estar totalmente de acuerdo con el ítem 7: se proyecta hacia la comunidad promoviendo actividades de desarrollo ambiental; el ítem 8: se promueven procesos de protección en el ambiente laboral; pero además el ítem 9: se ejecutan procesos para apoyar a las comunidades. Asimismo, el $20 \%$ consideró la alternativa totalmente en desacuerdo, un $7 \%$ estuvo de acuerdo, $6 \%$ se sentía indiferente mientras que un 5,1\% en desacuerdo, el promedio del indicador fue de 3,9. De este modo el indicador mejor entorno, el cual cuestiona con el ítem 10 si se le provee de los recursos materiales para ejecutar con eficiencia su trabajo, con el ítem 11 si el empleado recibe capacitación continua para ejecutar sus actividades laborales y con el ítem 12 si tiene a su disposición herramientas necesarias para ejecutar su trabajo.

Los datos arrojan los siguientes resultados: un $22,2 \%$ consideró estar indiferente, con respecto a las alternativas totalmente de acuerdo, de acuerdo y en desacuerdo, los valores fueron de $21,3 \%$ para cada una, asimismo el 5,1\% afirmaron estar totalmente en desacuerdo. Su promedio 3,2. Con respecto a la posesión de recursos, esto no se cumple, dado que el $36 \%$ seleccionó la alternativa totalmente en desacuerdo, un $32 \%$ considero estar indiferente, un 15,3\% estuvo de acuerdo, el $9 \%$ asumió la alternativa totalmente de acuerdo, y el restante $9 \%$ optó por estar en desacuerdo. El promedio del indicador se situó en 2,5. Estos datos son respuesta al ítem 13 que cuestiona si la empresa utiliza los recursos adecuados para la protección física del empleado, al ítem 14 sobre si se apoyan los proyectos para beneficiar a las comunidades, y con el ítem 15 se les planteó si el talento humano se involucra con proyectos de apoyo a las comunidades.

Para Certo, et al., (2007), la responsabilidad social es obligación de un individuo de manera particular, o puede ser tarea de un grupo de individuos que conforman una empresa, así Robbins y Coulter (2015), enuncian que, en el caso de muchos negocios, sus acciones sociales se consideran más como de sensibilidad social que de responsabilidad social. Además, asumen que parece ser que las acciones sociales de una empresa no lastiman su desempeño económico. Para establecer la relación entre calidad de servicio y responsabilidad social en empresas del sector logísticos, se aplicó el Coeficiente de Correlación a través del método de Spearman, para ello se computaron los datos arrojados designando la letra "X" para la variable calidad de servicio y la letra "Y" para la variable Responsabilidad Social. Esto se aprecia a mejor como:

$$
\begin{aligned}
& \sum R g s X=1275 \\
& \sum R g s Y=1275 \\
& \sum R g s \neq 0 \\
& \sum(X-Y)^{2}=16548
\end{aligned}
$$

El procedimiento fue ejecutado en el programa SPSS versión XXII, en tal sentido el cálculo de coeficiente de Spearman, arrojó un resultado de correlación de 0.205 , lo que indica la existencia de una correlación positiva. Es decir, la calidad de servicio tiene relación con la responsabilidad social, existiendo una relación entre ambas variables; asimismo se afirma que su dirección es directamente proporcional, es decir en el mismo sentido; la magnitud o intensidad es baja, sin embargo, indican que la responsabilidad social se relaciona con la calidad de servicio ofrecida.

\section{DISCUSIÓN}

Con respecto las dimensiones de calidad de servicio en empresa logísticas, se pudo conocer que el mobiliario es apropiado para la atención al cliente, la decoración de las oficinas se considera agradable y la apariencia física que promueve la buena imagen de la organización. Por otra parte, los productos ofrecidos por las oficinas son totalmente confiables, el servicio en las empresas es de satisfacción al cliente, así como 
también el cliente recibe constantemente los servicios requeridos. Las empresas del sector logístico, cuenta con el personal capacitado para satisfacer las necesidades del cliente, asimismo posee la cantidad adecuada de empleados para satisfacer las necesidades de sus clientes, asimismo al cliente se le suministra los servicios adecuadamente.

El nivel de seguridad que tiene el cliente respecto al servicio es medio, en ese sentido el cliente recibe respuestas acordes al servicio solicitado, además si el servicio ofrecido por las empresas genera seguridad al cliente, aunado al hecho de contar con un personal que le brinda confianza al cliente. Seguidamente se escuchan las sugerencias de los clientes, los empleados siempre están dispuestos a ofrecerles ayuda e información, la cortesía es base para la atención al cliente de las oficinas, ello incide en un mejor servicio. En cuanto a la responsabilidad social, se pudo corroborar que las expectativas públicas se están ejecutando cabalmente puesto que la empresa brinda la seguridad en cuanto a interés por servirle de una manera incondicional, asimismo, se ejecutan actividades para fomentar el proceso de integración del empleado, buscando realizar campañas para mejorar su entorno. En este ámbito la mayoría de la población consideró que esto se ejecuta en las Pymes

Además, las obligaciones éticas, permiten verificar la ejecución de acciones para el crecimiento personal del empleado, asimismo se percibe la mejora de los espacios físicos en el lugar de trabajo, además si se orientan actividades laborales, en función de la calidad de vida del empleado. Por otra parte, se concluye, que las empresas del sector logístico, no utiliza los recursos adecuados para la protección física del empleado, tampoco se apoyan los proyectos para beneficiar a las comunidades que están en sus áreas de interés, aunque sean un porcentaje mínimo, por ende, sus trabajadores no se involucran con proyectos de apoyo a las comunidades.

\section{CONCLUSIONES}

De acuerdo con el trabajo presentado y a los resultados obtenidos, se pueden plantear las siguientes conclusiones principales:

1. Los elementos de la calidad de servicio ofrecen alternativas a las empresas para visionar los procesos de atención al cliente, puesto que estas se sustentan en las expectativas y las necesidades del cliente, observando que los aspectos relacionados en esta investigación permiten verificar que en las empresas del sector logístico se activan los procesos requeridos para lograr un proceso confiable y coherente al cliente, ofreciendo espacios adecuados y personal integrado, además de conocedor de todas las actividades inherentes a los objetivos. Los empleados de estas empresas han considerado que el trabajo ejecutado se genera en función de ofrecer un servicio de alta calidad al cliente, donde ellos encuentren lo que necesitan y cumplan sus expectativas

2. En cuanto a la responsabilidad social, se evidencia que este proceso gerencial tan importante se desarrolla sustentado en Expectativas públicas, Obligación ética, Imagen pública, Mejor entorno y posesión de recursos, destacando que están orientadas a la búsqueda de acciones de responsabilidad social tanto interna como externa, dado que se realizan actividades para promover al personal, existen índices confiables de permanencia y baja rotación, se ejecutan actividades para fomentar el proceso de integración del empleado. Sin embargo, se destaca que no utiliza los recursos adecuados para la protección física del empleado, tampoco se apoyan los proyectos para beneficiar a las comunidades que están en sus áreas de interés, aunque sean un porcentaje mínimo, por ende, sus trabajadores no se involucran con proyectos de apoyo a las comunidades.

3. Para toda empresa es importante que se promueva un alto nivel en la calidad de servicio al cliente en este sentido se involucra al cliente interno al abarcar espacios y condiciones adecuadas para atención al cliente externo. Cuando un cliente asiste a solicitar un servicio llega con necesidades y expectativas, y es el empleado quien da la primera imagen al cliente, destacando la posibilidad de promover y generar satisfacción en el servicio. Asimismo, el hecho de cubrir las necesidades del cliente considerando los aspectos normativos, ergonómicos y de personal preparado e integrado a la filosofía de gestión asocia un proceso de responsabilidad social.

4. Para una empresa ser responsable socialmente no es solo mirar quien está a su alrededor, sino comenzar un proceso interno, de integración y respeto al empleado, generando acciones positivas al cliente, lo cual vincula imagen, relación con el entorno, así como las obligaciones sociales.

\section{REFERENCIAS}

Bravo, S., Donado, A., y otros 3 autores, MiPyMEs asociadas y no asociadas: una aproximación a su gestión en innovación y desarrollo tecnológico, https://www.revistaespacios.com/a17v38n58/a17v38n58p17.pdf, ISSN: 0798-1015, Revista Espacios, 38(58), 17-37 (2017) 
Certo, S., Holmes, M., y Holcomb, R., The influence of people on the performance of IPO firms. Business Horizons, 50(4), 271-276 (2007)

De la Hoz, J., Hernández, H. y otros 6 autores, Management model for the logistics and competitiveness of SMEs in the city of Barranquilla. In: Rocha, Á., Reis, J., Peter, M., y Bogdanović, Z., (eds) Marketing and smart technologies, doi: https://doi.org/10.1007/978-981-15-1564-4_37, Smart Innovation, Systems and Technologies, v. 167. Springer, (2020)

Díaz, Y., Andrade, J. y Ramírez, E., Liderazgo transformacional y responsabilidad social en asociaciones de mujeres cafeteras en el sur de Colombia, doi: https://dx.doi.org/10.4067/S0718-07642019000500121, Inf. Tecnol., 30(5), 121-130, (2019)

Díaz, J., Ruiz, J., Contreras, J. y Hernández, H, Technology management to increase the efficiency of the supply chain. http://www.jatit.org/volumes/Vol95No19/30Vol95No19.pdf, ISSN: 1992-8645, Journal of Theoretical and Applied Information Technology, 95(19), (2017)

Durán, S., García, J., Crissien, J. y Virviesca, J., Estrategias gerenciales para la formación de equipos de trabajo en empresas constructoras del Caribe Colombiano, https://www.revistaespacios.com/a17v38n13/a17v38n13p24.pdf, ISSN: 0798-1015, Revista Espacios, 38(13), 24-39 (2017)

Unión Europea, Libro verde de la Comisión Europea, Barcelona: Estudi6 Barcelona (2002)

García, J., Durán, S., y otros 4 autores, Proceso de planificación estratégica: etapas ejecutadas en pequeñas y medianas empresas para optimizar la competitividad. https://www.revistaespacios.com/a17v38n52/a17v38n52p16.pdf, ISSN: 0798-1015, Revista Espacios, 38(52), 16-30 (2017)

García, J., Durán, S., Parra, M., y Márceles, V., Dirección estratégica del talento humano para el fomento de valores en los cuerpos policiales venezolanos, https://www.revistaespacios.com/a17v38n32/a17v38n32p16.pdf, ISSN: 0798-1015, Revista Espacios, 38(32), 16-28 (2017)

Jiménez, J., Mojica, J., Hernández, H., y Cardona, D., Diagnóstico de la innovación y desarrollo tecnológico en el sector hotelero de la región caribe colombiana, doi: https://dx.doi.org/10.4067/S0718-07642018000500157, Información Tecnológica, 29(5), 157-164 (2018)

Jiménez, G., Hernández, L., y otros 3 autores, Evaluation of quality management for strategic decision making in companies in the plastic sector of the Colombian Caribbean region using the TQM diagnostic report and data analysis. In: Stephanidis C. (eds) HCl International 2018 - Posters' Extended Abstracts. HCl 2018. Communications in Computer and Information Science, doi: https://doi.org/10.1007/978-3-319-92285-0_38, Springer, Cham, Vol 852 (2018)

Kerin, R., y Hartley, S., Marketing: the core. McGraw-Hill (2015)

Kotler, P., Principles of marketing. Pearson higher education (2017)

Koontz, H., y Weihrich, H., Management: A global perspective. Tata McGraw-Hill (2012)

Koontz, H., y Weihrich, H., Essentials of management: an international, innovation, and leadership perspective. McGrawHill Education, (2015)

Martínez, J., Cardeño, E., y otros 2 autores, Liderazgo transformacional como estrategia de adaptación en la gestión logística empresarial, doi: https://doi.org/10.17081/dege.9.2.2980, Rev. Desarrollo Gerencial, 9(2), 140-157 (2017)

Moreno, M., Tezón, M., y otros 3 autores., Autoestima: desarrollo de la autonomía personal en estudiantes del área técnica, ISSN: 0798-1015, Revista Espacios, 39(46), 6-11 (2018)

Padilla, C., Arévalo, D., y otros 3 autores., Responsabilidad social empresarial y desempeño financiero en la industria del plástico en ecuador, doi: https://dx.doi.org/10.4067/S0718-07642017000400012, Inf. Tecnol., 28(4), 93-102 (2017)

Parra, M., Visbal, O., Durán, S., y Badde, G., Calidad de la comunicación y actitud de los empleados ante procesos de cambio organizacional Interdisciplinaria, doi: https://doi.org/10.16888/interd.2019.36.1.11, Centro Interamericano de Investigaciones Psicológicas y Ciencias Afines, 36 (1), 155-170 (2019)

Parody, A., Viloria, A., y otros 4 autores (Application of an Experimental Design of D-Optimum Mixing Based on Restrictions for the Optimization of the Pre-painted Steel Line of a Steel Producer and Marketing Company. In: Tan, Y., Shi, Y., y Tang Q., (eds) Data Mining and Big Data. DMBD 2018. Lecture Notes in Computer Science, vol 10943 . Springer, Cham. (2018)

Paz, A., Harris, J., y García, J., Toma de decisiones: reto para crear ventajas competitivas en las distribuidoras de alimentos gourmet, doi: http://dx.doi.org/10.17081/dege.7.2.1183, Revista Desarrollo Gerencial, 7(2), 100-118 (2015)

Perreault, W., Cannon, J., y McCarthy, E., Basic marketing: a marketing strategy planning approach. $19^{\text {th }}$ Edition. McGraw-Hill Education, (2013)

Pride, W., y Ferrell, O.C., Foundations of marketing. $8^{\text {th }}$ Edition. Cengage Learning (2018)

Robbins, S., y Coulter, M., Management, Global Edition. Pearson Education Limited (2015)

Robbins, S., y Judge T., Organizational Behavior, 17th Edition, Pearson Editorial Pearson (2017)

Stanton, W., Etzel, M., y Walker, B., Marketing, McGrawHill. New York. USA (2012)

Zeithaml, B., Bitner, M., y Gremler, D., Service Marketing: Integrating customer focus across the firm, $7^{\text {th }}$ edition, McGraw Hill (2017) 
